\title{
ANTECEDENTES DEL MERCADO INTERNACIONAL DE PIÑONES
}

DE PINO. Daniel Soto A., Janina Gysling C., Verónica Loewe M. Ingenieros Forestales Universidad de Chile, Investigadores Instituto Forestal. dsoto@infor.cl; jgysling@infor.cl; vloewe@infor.cl, Sucre 2397, Santiago. Chile.

\section{RESUMEN}

El Instituto Forestal está desarrollando trabajos de investigación sobre las posibilidades de cultivo de pino piñonero (Pinus pinea) en el pais y producción y comercialización de sus frutos. En el presente trabajo se analizan los volúmenes de producción mundial de frutos secos, las características del fruto de diferentes especies del género Pinus que producen piñones, los principales mercados externos y el local, los estándares de calidad en los mercados internacionales, los canales de comercialización y los precios.

Se revisa igualmente las zonas potenciales para el establecimiento de plantaciones de esta especie en el pais, principalmente en las Regiones de O'Higgins y Maule, en donde en las áreas de secano interior de condiciones semiáridas, la especie podria representar una interesante oportunidad de innovación y diversificación productiva.

Palabras chave: Pinus pinea, mercado, piñones.

\section{SUMMARY}

Research programs on the possibilities to cultivate Pinus pinea in Chile to produce and market Pine nuts, are being carried out by the Forest Institute. This paper analyses different dry fruits world production volumes, main characteristics of fruits from several Pinus species producing nuts, main international markets and the local one, quality standards in the international market, market channels and prices.

Potential areas in the country to establish planted forest with the species are reviewed as well, mainly dry interior zones in the O'Higgins and Maule Regions, where the species could represent an interesting and innovative way for production diversification.

Key words: Pinus pinea, market, Pine nuts

\section{INTRODUCCIÓN}

La denominación de fruto seco se refiere a una semilla comestible, pobre en agua y rica en aceite o en almidón, la que está encerrada en una cáscara dura. Los frutos secos pueden ser: Oleaginosos; semillas ricas en aceite, entre los que se incluyen las nueces, almendras, avellanas y mani; y Farináceos; semillas que contienen almidón en abundancia, como la castaña, el piñón y otros. 
El fruto seco es la semilla de un fruto, y no su parte carnosa, que suele no ser comestible. La mayor parte de los frutos secos proceden de árboles que no pertenecen a la familia de las leguminosas, sin embargo, el mani es la excepción, ya que no crece en un árbol, sino bajo tierra. Por su riqueza en aceite, la semilla de mani no se incluye entre las legumbres, sino entre los frutos secos. Los frutos secos no deben confundirse con la fruta dulce desecada por diversos procedimientos (pasas, ciruelas secas, etc.) pues su parte comestible es el pericarpio (pulpa) y no la semilla.

\section{Cuadro $\mathrm{N}^{\circ} 1$ \\ EVOLUCIÓN DE LA PRODUCCIÓN MUNDIAL DE LOS PRINCIPALES FRUTOS SECOS CON CÁSCARA (toneladas)}

\begin{tabular}{|c|c|c|c|c|c|}
\hline Fruto & $\begin{array}{c}\text { Media } \\
1983- \\
1986\end{array}$ & $\begin{array}{c}\text { Media } \\
1987- \\
1990\end{array}$ & $\begin{array}{c}\text { Media } \\
1991- \\
1994\end{array}$ & $\begin{array}{c}\text { Media } \\
1994- \\
1998\end{array}$ & $\begin{array}{l}\text { Media } \\
1999- \\
2002\end{array}$ \\
\hline $\begin{array}{l}\text { Almendra } \\
\text { Prunus amygdalus Batsch (Sin: } \\
\text { Prunus dulcis (Miller)) } \\
\text { Fam: Rosaceae }\end{array}$ & 1.040 .926 & 1.251 .288 & 1.298 .331 & 1.292 .437 & 1.558 .521 \\
\hline $\begin{array}{l}\text { Anacardo } \\
\text { Anacardium occidentale } L \text {. } \\
\text { Fam: Anacardiaceae }\end{array}$ & 541.698 & 691.100 & 930.707 & 1.251 .938 & 1.556 .057 \\
\hline $\begin{array}{l}\text { Avellana } \\
\text { Corylus avellana } L . \\
\text { Fam. Corylaceae }\end{array}$ & 470.032 & 607.297 & 617.503 & 682.819 & 784.730 \\
\hline $\begin{array}{l}\text { Nuez } \\
\text { Juglans regia L. } \\
\text { Fam: Juglandaceae }\end{array}$ & 837.719 & 884.656 & 1.001 .174 & 1.103 .483 & 1.249 .711 \\
\hline $\begin{array}{l}\text { Nuez de Brasil } \\
\text { Berholletia excelsa Humb. et } \\
\text { Bonpl. } \\
\text { Fam: Lecythidaceae }\end{array}$ & 63.149 & 59.294 & 55.933 & 51.033 & 69.920 \\
\hline $\begin{array}{l}\text { Pistacho } \\
\text { Pistacia vera } L \text {. } \\
\text { Fam: Anacardiaceae }\end{array}$ & 192.547 & 235.025 & 354.853 & 420.559 & 472.794 \\
\hline $\begin{array}{l}\text { Macadamia } \\
\text { Macadamia integrifolia Maiden et } \\
\text { Betche (cáscara lisa) } \\
\text { Macadamia tetraphilla L.A.S. } \\
\text { Johnson (cáscara rugosa) } \\
\text { Fam: Proteaceae }\end{array}$ & nd & nd & nd & nd & 89.803 \\
\hline $\begin{array}{l}\text { Pecana } \\
\text { Carya illinoiensis (Wang) K. Kosh } \\
\text { Fam: Juglandaceae }\end{array}$ & nd & nd & nd & nd & 187.811 \\
\hline $\begin{array}{l}\text { Piñón } \\
\text { Pinus pinea Linneo } \\
\text { Fam: Pinaceae }\end{array}$ & nd & nd & nd & nd & 25.000 \\
\hline
\end{tabular}

nd: información no disponible. Fuente: (Salas-Salvadó et al., 2005). 
Hasta hace unos años, muchos nutricionistas pensaban que los frutos secos oleaginosos eran nocivos para la salud del corazón y de las arterias, debido a su elevada proporción de grasa. Actualmente, han dejado de ser considerados por la mayor parte de los especialistas como enemigos del corazón, pues diversas investigaciones confirman su acción protectora de las arterias y reductora del nivel de colesterol.

Los frutos secos tienen caracteristicas en común, como sus propiedades fisicas, químicas y nutritivas, asi como la recolección, post cosecha, procesamiento y comercialización de los productos también tienen muchos aspectos similares. Botánicamente hay algunas especies similares (por ejemplo, nogal y pecano), otras se acercan de acuerdo a sus requerimientos climáticos, por ejemplo el anacardo y el nogal de Brasil requieren climas cálidos, mientras el pistachero y el pino piñonero son de climas mediterráneos. En relación a la tolerancia a la sequía se asemejan el almendro, el pino piñonero y el pistachero, por sus bajos requerimientos, en tanto que anacardo y macadamia producen en suelos más húmedos.

La producción de frutos secos se obtiene principalmente de plantaciones regulares en almendros, avellanos, macadamias, nogales, pecanos y pistacheros; de plantaciones y árboles silvestres en anacardos; y de bosques naturales en nogales de Brasil y piñón europeo.

\section{CARACTERÍSTICAS DE LOS PIÑONES DEL PINO}

Los piñones constituyen un producto forestal no maderero, cosechados en varias regiones del mundo, en bosques naturales y plantaciones. Su consumo como alimento data de los tiempos de las antiguas Roma y Grecia, y han sido un componente muy importante de la alimentación de diferentes tribus indigenas de América, Siberia y el Lejano Oriente. Actualmente, los piñones continúan siendo cosechados, teniendo un gran reconocimiento como producto gourmet en los mercados internacionales.

Aproximadamente 29 especies de pinos producen semillas comestibles, al menos en culturas tribales, pero sólo algunos de ellos son importantes a nivel comercial (Cuadro $\mathrm{N}^{\circ} 2$ ).

En el Cuadro $\mathrm{N}^{\circ} 3$ se indica que los contenidos nutricionales de los piñones de Pinus pinea son muy interesantes, puesto que presentan los mayores valores de proteinas, los menores de carbohidratos y un valor bastante bajo de grasas, lo que le confiere grandes ventajas comerciales. Son considerados como productos libres de colesterol puesto que el $93 \%$ de su contenido oleico es de grasas no saturadas.

En efecto, los piñones son conocidos, a través del mundo por sus saludables propiedades nutricionales en estado crudo o tostado. Es un ingrediente esencial en múltiples platos de la cocina oriental y mediterránea y, también, es muy utilizado en chocolateria y pasteleria.

Los piñones de las distintas especies de pinos difieren en tamaño, contenido nutricional, valor medicinal y gusto, sin embargo, la mayoria de los consumidores no son tan sofisticados como para distinguir entre piñones de diferentes especies y por esto hay que considerar que, en el ámbito comercial, el término "piñones" (en inglés: Pine nuts, pinyon nuts y pignolia, 
principalmente) ${ }^{1}$ se utiliza para todos los frutos de las especies del género Pinus, a menos que se haga expresa mención de la especie.

Por su alto contenido de grasa, sobre el $60 \%$, en algunos paises, como por ejemplo Rusia, los piñones también son utilizados para la fabricación de aceite, bajo un proceso de prensado. El aceite resultante participa en el nicho de productos gourmet de alto valor o se vende como medicina en botellas o cápsulas. También es usado en cosméticos y productos de belleza, y en la industria de pinturas y barnices. Cabe señalar que como subproducto del proceso de prensado para aceite, se obtiene las hojuelas de piñones que se usan en chocolates, cereales y otros productos para snacks.

La caracteristica de los piñones que más se explota a nivel comercial es la elevada concentración de grasas monoinsaturadas, que fomentan un sistema cardiovascular sano. La vitamina $\mathrm{D}$ contribuye a la formación de huesos y dientes resistentes, mejorando la habilidad de fijar el calcio; también las vitaminas A y C pueden mejorar la visión y robustecer el sistema inmunitario.

Las principales especies productoras de piñones con importancia comercial mundial son 1) Pinus pinea (piñón europeo, Italian Stone Pine); 2) Pinus sibirica (pino de Siberia); 3) Pinus koraiensis (pino de Corea); 4) Pinus gerardiana (Chilgoza Pine) y 5) Pinus monophylla (piñón de aguja sencilla), Pinus edulis (piñón del Colorado) y otras especies de piñones (Sharashkin y Gold, 2004).

Existen distinciones entre los piñones producidos en diferentes áreas geográficas. El piñón de Europa se denomina "pignolia", un término que se refiere al piñón del pino piñonero (Pinus pinea) que crece en España, Portugal, Italia y Norte de África. Los piñones de una especie diferente corresponden a los "pinyon", nombre derivado de la palabra española y que se produce en el oeste de EEUU; estos piñones vienen principalmente del pino piñonero del Colorado, un pino de dos acículas que crece en forma silvestre en los estados de Colorado, Nuevo México, Arizona, y Utah.

'Piniennufs (alemán), pinnoli (italiano), pinhào (portugés), pignólia (griego), song guo (chino). 


\section{Cuadro $\mathrm{N}^{\circ} 2$ \\ ESPECIES DE PINOS CON PIÑONES COMESTIBLES}

(FAO, 2005)

\begin{tabular}{|c|c|c|}
\hline Especies & $\begin{array}{c}\text { Rango de distribución } \\
\text { natural }\end{array}$ & Importancia económica \\
\hline \multicolumn{3}{|c|}{ Pinos Haploxylon (blandos) } \\
\hline P. ayacahuite & México, Central América & $\begin{array}{l}\text { Alimento tradicional para } \\
\text { tribus indigenas. }\end{array}$ \\
\hline P. albicaulis & Oeste de Canadá y EEUU & $\begin{array}{l}\text { Alimento tradicional para } \\
\text { tribus indigenas. }\end{array}$ \\
\hline P. cembra & $\begin{array}{c}\text { Europa (Alpes y los } \\
\text { Cárpatos) }\end{array}$ & $\begin{array}{l}\text { Localmente importante. } \\
\text { Piñones de bajo valor.. }\end{array}$ \\
\hline P. flexilis & Oeste de Canadá y EEUU & $\begin{array}{l}\text { Alimento tradicional para } \\
\text { tribus indigenas. }\end{array}$ \\
\hline P. gerardiana & Afganistán, Pakistán, India & $\begin{array}{c}\text { Importante en el comercio } \\
\text { internacional. }\end{array}$ \\
\hline P. koraiensis & China, Japón, Corea, Liberia & $\begin{array}{l}\text { Importante en el comercio } \\
\text { internacional. }\end{array}$ \\
\hline P. lambertiana & $\begin{array}{l}\text { Oeste de EEUU (California, } \\
\text { Oregón) }\end{array}$ & $\begin{array}{l}\text { Alimento tradicional para } \\
\text { tribus indigenas. }\end{array}$ \\
\hline P. monticola & $\begin{array}{c}\text { EEUU y zonas limitrofes de } \\
\text { Canadá }\end{array}$ & $\begin{array}{c}\text { Alimento tradicional para } \\
\text { tribus indigenas. }\end{array}$ \\
\hline P. pumila & $\begin{array}{l}\text { Siberia, China, Corea, } \\
\text { Japón }\end{array}$ & $\begin{array}{c}\text { Valor económico intermedio, } \\
\text { buena cosecha pero } \\
\text { piñones pequeños. }\end{array}$ \\
\hline P. sibirica & $\begin{array}{c}\text { Rusia (Siberia Central), } \\
\text { Mongolia }\end{array}$ & $\begin{array}{l}\text { Alto valor económico del } \\
\text { piñón. También es cultivada } \\
\text { para madera. }\end{array}$ \\
\hline Pinos piñoneros & $\begin{array}{c}\text { Varias especies en México } \\
\text { y EEUU }\end{array}$ & \\
\hline \multicolumn{3}{|c|}{ Pinos Diploxylon (duros) } \\
\hline P. coulteri & EEUU (California) & $\begin{array}{l}\text { Alimento tradicional para } \\
\text { tribus indigenas. }\end{array}$ \\
\hline P. pinea & $\begin{array}{l}\text { Europa Mediterránea y } \\
\text { cercano oriente }\end{array}$ & $\begin{array}{l}\text { Importante en el comercio } \\
\text { internacional. }\end{array}$ \\
\hline P. ponderosa & Oeste de Canadá y EEUU & $\begin{array}{l}\text { Alimento tradicional para } \\
\text { tribus indigenas. }\end{array}$ \\
\hline P. sabiniana & EEUU (California) & $\begin{array}{l}\text { Alimento tradicional para } \\
\text { tribus indigenas. }\end{array}$ \\
\hline P. roxburghii & India & $\begin{array}{l}\text { Fuente de alimento } \\
\text { tradicional. }\end{array}$ \\
\hline P. torreyana & EEUU (California) & $\begin{array}{l}\text { Alimento tradicional para } \\
\text { tribus indigenas. }\end{array}$ \\
\hline
\end{tabular}




\section{Cuadro $\mathrm{N}^{\circ} 3$ \\ VALOR NUTRICIONAL DE DISTINTOS PIÑONES Y DE OTROS FRUTOS SECOS COMERCIALIZADOS}

(FAO, 2005)

\begin{tabular}{|l|c|c|c|}
\hline Tipo de Nuez & Proteinas (\%) & Grasas (\%) & $\begin{array}{c}\text { Carbohidratos } \\
\text { (\%) }\end{array}$ \\
\hline Pinus edulis & 14 & $62-71$ & 18 \\
\hline Pinus monophylla & 10 & 23 & 54 \\
\hline Pinus cembroides & 19 & 60 & 14 \\
\hline Pinus quadrifolia & 11 & 37 & 44 \\
\hline Pinus sabiniana & 30 & 60 & 7 \\
\hline Pinus strobiformis & 28 & 52 & 7 \\
\hline Pinus pinea & 34 & 48 & 12 \\
\hline Pinus sibirica & 19 & $51-75$ & 23 \\
\hline Pinus gerardiana & 14 & 51 & 11 \\
\hline Pecano (Carya illinoiensis) & 10 & 73 & 24 \\
\hline Mani (Arachis hypogaea) & 26 & 39 & 12 \\
\hline Nuez común (Juglans regia) & 15 & 68 & 9 \\
\hline
\end{tabular}

También existen diferentes variedades de pinos piñoneros que crecen en Rusia, Corea, Japón y China. En EEUU el término "piñón" puede referirse al piñón europeo, al piñón norteamericano o al chino, que tambièn se produce en Corea y que corresponde a la especie Pinus koraiensis. En Pakistán y la India se cosechan piñones del Pinus gerardiana, bien conocido por sus semillas comestibles y localmente se le comercializa como "chilgoza". En la India, constituye uno de los más importantes cultivos de las tribus residentes en el distrito de Kinnaur.

A pesar de que el piñón europeo puede ser comido directamente, crudo o tostado, se caracteriza por ser el único usado en gastronomia; en Europa se usa asociado a carnes. pescados y aves, y en múltiples salsas. 


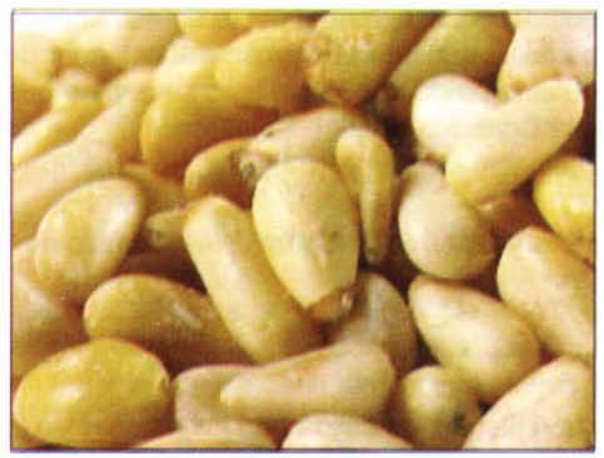

Figura $\mathrm{N}^{\circ} 1$

PIÑONES DEL PINO PIÑONERO

EUROPEO

(Fuente: http://www.nutsonline.com/nuts/pinenuts/ pine.htm)

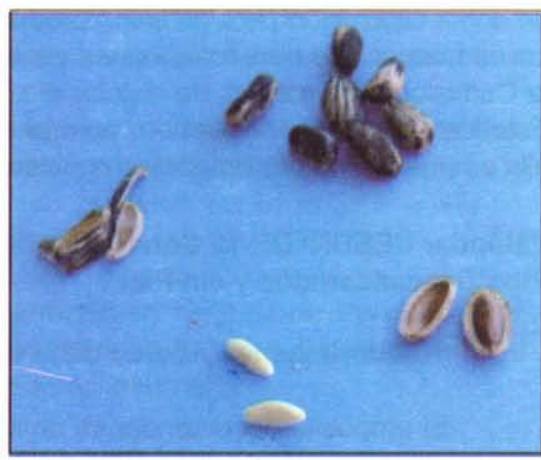

Figura $\mathrm{N}^{\circ} 2$

PIÑONES DEL PINO PIÑONERO

EUROPEO EN DIFERENTES

ETAPAS

(Fuente: Wikipedia, 2008).

El árbol del piñón norteamericano tiene inferiores caracteristicas que el europeo, que posee una mejor madera, alcanza mayor tamaño y crece más rápido. Las plantaciones de pino piñonero europeo que se han establecido en la zona del Mediterráneo se mantienen bien desarrolladas, mientras que el piñón americano ha tenido escasa atención y prácticamente no ha sido cultivado. En relación a la semilla, es más frecuente encontrar el piñón chino en el mercado de EEUU, debido a su mayor disponibilidad y a su precio más accesible. Los piñones chinos y europeos se comercializan pelados, ya listos para su uso.

\section{ESTÁNDARES DE CALIDAD PARA EL COMERCIO INTERNACIONAL DE PIÑONES DE PINO EN EUROPA Y ESTADOS UNIDOS}

La Comisión Económica de las Naciones Unidas para Europa definió en 1993 un estándar de control de calidad para el piñón de pino, tanto para el comercio intra como extra europeo. Este estándar opera a nivel de recomendación a los paises de esta Comisión, la cual insta a sus miembros a utilizarlo como línea de base para facilitar el acceso a los mercados por parte de los diversos actores, al mismo tiempo que protege los intereses de los consumidores. Sin embargo, en el marco de la normativa vigente en la Unión Europea, no existe un reglamento especifico que regule el comercio del piñón del pino en este mercado.

En el documento de la Comisión se define la aplicación de un estándar a las semillas del género Pinus spp., en especial a los piñones de la especie Pinus pinea L. y al piñón chino (Pinus koraiensis), en las cuales el endocarpio (cáscara) y el tegumento (piel) han sido removidos. El estándar se aplica a los piñones tanto de consumo directo como para los destinados a procesamiento industrial.

Por su parte, en Estados Unidos, de acuerdo con el Servicio de Comercialización Agrícola, del Departamento de Agricultura, organismo encargado de emitir estándares y órdenes 
de comercialización para diversos productos agricolas, no existe formalmente un estándar de calidad especifico para los piñones del pino, sin perjuicio que se deba recurrir al estándar de la Comisión para efectos de regular el comercio de este producto. Esto último se debe a que Estados Unidos es un miembro permanente de esta Comisión y todas las normativas que de ella emanen deben aplicarse a sus paises integrantes.

\section{Estándar CEEUN² DF-12 Concerniente al Comercio y Control de Calidad de Piñones de Pino Descascarados y sin Piel}

\section{-Requerimientos Acerca de la Calidad}

El propósito del estándar es definir los requerimientos de calidad de los piñones en la etapa de exportación posterior a su preparación y embalaje. Para ello se ha establecido los requerimientos minimos que deben cumplir todos los tipos de piñones y el contenido de humedad.

\section{a. Requerimientos mínimos}

Aplicable a todos los piñones, los que deben ser:

\section{Enteros.}

Sanos: Se excluyen aquellos afectados por pudrición o defectos que los hacen no aptos para el consumo.

Suficientemente desarrollados: Sin indicios de germinación.

Limpios, cepillados, lavados: Prácticamente libres de sustancias extrañas, incluyendo partes de piel de la semilla y de cáscara.

Libre de insectos vivos o ácaros en cualquiera de sus etapas de desarrollo.

Libre de daño visible por insectos, ácaros u otros parásitos.

Libre de moho.

Libre de fermentación y ranciedad.

Libre de humedad externa anormal.

Libre de olores y sabores anormales.

Sobre el contenido de humedad:

Los piñones no deben contener más de $6 \%$ de contenido de humedad, siendo éste determinado por métodos que se detallan en el anexo del estándar.

\section{b. Clasificación}

Clase Extra. Los piñones deben ser de calidad superior, ser caracteristicos de las especies o tipos comerciales y ser de color uniforme. Deben estar prácticamente libres de defectos y manchas, con excepción de defectos superficiales muy leves, tales que no afecten la apariencia general del producto, su calidad o su conservación en el envase.

${ }^{2}$ CEEUN : Comisión Económica de las Naciones Unidas para Europa. 
Clase I. Los piñones deben ser de buena calidad y caracteristicos de las especies y/o tipos comerciales. Pueden tener los siguientes defectos en forma leve: En la forma, en su desarrollo y en la coloración.

Clase II. Esta clase incluye los piñones que no clasifican en las clases anteriores, pero que satisfacen los requerimientos minimos expresados anteriormente.

\section{-Requerimientos Acerca del Tamaño}

Los piñones no requieren ser clasificados por tamaño en ninguna de las clases, sin embargo, ellos pueden presentarse graduados por tamaño en base al mayor diámetro transversal, expresados en milimetros o fracción de milimetros.

\section{-Requerimientos Acerca de las Tolerancias}

\section{a. Tolerancias en Calidad}

Se permitirán tolerancias en la calidad cuando no se cumpla con los requerimientos de calidad de la clase especificada.

\section{Cuadro $\mathrm{N}^{\circ} 4$}

\section{TOLERANCIAS SOBRE DEFECTOS PERMITIDOS PARA EL COMERCIO EUROPEO DE} PIÑONES

\begin{tabular}{|c|c|c|c|}
\hline \multirow{2}{*}{ Defectos permitidos } & \multicolumn{3}{|c|}{ Porcentaje del peso $(\%)$} \\
\hline & Extra & Clase I & Clase II \\
\hline Tolerancia total. & 5 & 10 & 15 \\
\hline Defectos especificos. & - & - & - \\
\hline $\begin{array}{l}\text { Piñones excesivamente secados o poco } \\
\text { desarrollados. }\end{array}$ & 1 & 3 & 5 \\
\hline Piñones en etapa de germinación. & 1 & 2 & 4 \\
\hline Piñones fermentados o rancios. & 0,2 & 0,5 & 1 \\
\hline Piñones podridos o mohosos ${ }^{3}$. & 0,2 & 0,4 & 0,8 \\
\hline Piñones dañados por insectos ${ }^{3}$. & 0,2 & 0,4 & 0,8 \\
\hline Piñones aplastados, quebrados o trozados. & 3 & 6 & 10 \\
\hline $\begin{array}{c}\text { Piñones con defectos superficiales o trazas de } \\
\text { tegumento. }\end{array}$ & 2 & 4 & 6 \\
\hline $\begin{array}{l}\text { Materiales vegetales extraños (cáscara, piel, polvo, } \\
\text { etc.). }\end{array}$ & 0,2 & 0,3 & 0,5 \\
\hline
\end{tabular}

\footnotetext{
${ }^{3}$ Alemania y Suiza no aceptan tolerancias para el producto afectado por moho o pudrición, o la presencia de insectos vivos o muertos.
} 


\section{b. Tolerancias en el tamaño}

Si los piñones son presentados por grados de tamaño, se acepta hasta un $20 \%$ de piñones fuera del tamaño señalado.

\section{- Requerimientos Acerca de la Presentación}

Uniformidad: El envase sólo debe contener piñones, del mismo origen y calidad. La parte visible del envase debe ser representativa de su total contenido.

Embalaje: Debe ofrecer entera protección a los piñones.

Presentación: Para todos los casos, los piñones bajo este estándar deben ser envasados en contenedores rígidos, con un peso neto no superior a $25 \mathrm{~kg}$, o en bolsas, con un peso neto no superior a $50 \mathrm{~kg}$.

- Requerimientos Acerca del Marketing

Se debe indicar como minimo: Identificación del producto, Naturaleza del producto, Origen del producto, Especificación comercial y Marca de la oficina de control (opcional).

\section{- Anexos del Estándar}

Los anexos del estándar comprenden las siguientes definiciones:

Anexo I. Determinación del contenido de humedad para producción de nueces * secas. Método 1. Mediante laboratorio, Método 2. Método rápido.

\section{ANTECEDENTES DEL MERCADO INTERNACIONAL}

\section{Estados Unidos}

Aunque no se dispone de cifras a nivel mundial como para hacer una comparación por paises, la bibliografia destaca la supremacia de EEUU como consumidor e importador de piñones y productos de piñones. En este mercado el término piñón puede referirse al piñón europeo, al asiático o al de otras especies, excepto si se menciona la especie.

De acuerdo a antecedentes de la International Trade Administration (ITA) de EEUU, el consumo de piñones en este pais representa un mercado de unos US\$100 millones, con un importante potencial de crecimiento, si se considera que actualmente está poco desarrollado. Al respecto, los especialistas señalan que una de las limitantes al crecimiento del mercado es el hecho de que los piñones son considerados como un producto especial y se venden sólo, a través de distribuidores especializados, como tiendas de alimentos naturales, de productos

\footnotetext{
${ }^{4}$ Nombre genérico para frutos secos. Del inglés "nuts".
} 
étnicos, de productos para la salud y por catálogos, alcanzando altos precios.

Sin embargo, también el crecimiento del mercado está limitado por la oferta de piñones. Considerando un año de buena cosecha, se estima que la producción mundial de piñones puede alcanzar a 20 mil toneladas de piñones. No obstante, esta producción puede variar significativamente de un periodo a otro. Casi todos los años la oferta local de piñones se termina unos meses antes que se realice la nueva cosecha, lo que produce precios altos e inelásticos, es decir, aunque luego la oferta aumente significativamente, el precio no baja o la baja es casi imperceptible. Por otra parte, esta situación también refleja que no hay grandes sustitutos para los piñones (Sharashkin y Gold, 2004).

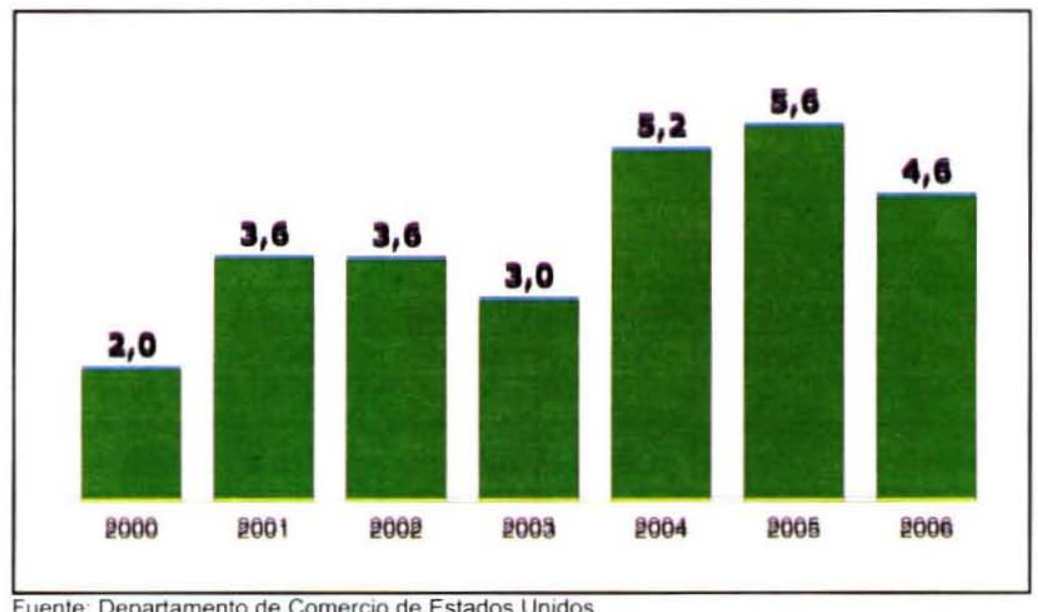

Fuente: Departamento de Comercio de Estados Unidos

HS: 080290.20 Y 080290.25 .

Figura $\mathrm{N}^{\circ} 3$

IMPORTACIONES DE PIÑONES EN ESTADOS UNIDOS (miles de toneladas)

EEUU suple la falta de oferta local con importaciones, tanto que entre el $80 \%$ y el $90 \%$ de su consumo proviene del exterior. Durante el año 2006, las importaciones de piñones alcanzaron a US\$ 51,4 millones, equivalentes a un volumen de 4.560 toneladas. De este volumen, el $96 \%$ corresponde a piñones secos o frescos pelados, mientras que el restante $4 \%$ corresponde a piñones secos o frescos sin pelar. 


\section{Cuadro $\mathrm{N}^{\circ} 5$ \\ IMPORTACIONES DE PIÑONES EN ESTADOS UNIDOS (toneladas)}

\begin{tabular}{|c|c|c|c|c|c|c|c|}
\hline Pais & 2000 & 2001 & 2002 & 2003 & 2004 & 2005 & 2006 \\
\hline \multicolumn{8}{|c|}{ 0802.9020 Piñones, frescos o secos, con cáscara } \\
\hline China & 41 & 113 & 16 & 135 & 1,051 & 791 & 76 \\
\hline Afganistán & 0 & 0 & 0 & 0 & 0 & 0 & 86 \\
\hline Turquia & 0 & 0 & 0 & 0 & 18 & 162 & 14 \\
\hline Italia & 5 & 0 & 0 & 0 & 0 & 0 & 0 \\
\hline Rusia & 3 & 0 & 0 & 0 & 0 & 0 & 0 \\
\hline Bolivia & 0 & 0 & 0 & 0 & 0 & 0 & 0 \\
\hline Holanda & 0 & 11 & 0 & 0 & 0 & 0 & 0 \\
\hline Pakistán & 3 & 1 & 7 & 0 & 3 & 0 & 0 \\
\hline España & 0 & 0 & 0 & 0 & 0 & 0 & 0 \\
\hline Subtotal & 52 & 125 & 23 & 135 & 1072 & 953 & 176 \\
\hline \multicolumn{8}{|c|}{ 0802.90.25 Piñones, frescos o secos, sin cáscara } \\
\hline China & 1.617 & 3.382 & 3.464 & 2.734 & 4.042 & 4.549 & 4.251 \\
\hline España & 28 & 46 & 50 & 36 & 39 & 27 & 31 \\
\hline $\begin{array}{l}\text { Hong } \\
\text { Kong }\end{array}$ & 141 & 15 & 35 & 0 & 11 & 42 & 51 \\
\hline Suiza & 0 & 0 & 0 & 0 & 7 & 0 & 30 \\
\hline Pakistán & 85 & 36 & 1 & 7 & 11 & 1 & 20 \\
\hline Turquia & 12 & 6 & 30 & 62 & 48 & 26 & 1 \\
\hline India & 0 & 0 & 0 & 0 & 0 & 0 & 0 \\
\hline Italia & 1 & 0 & 0 & 0 & 0 & 0 & 0 \\
\hline Canadá & 0 & 0 & 0 & 0 & 1 & 0 & 0 \\
\hline Rusia & 0 & 0 & 3 & 10 & 0 & 0 & 0 \\
\hline Portugal & 25 & 16 & 0 & 0 & 10 & 0 & 0 \\
\hline $\begin{array}{l}\text { Reino } \\
\text { Unido }\end{array}$ & 0 & 0 & 0 & 0 & 0 & 0 & 0 \\
\hline Holanda & 0 & 0 & 0 & 15 & 0 & 0 & 0 \\
\hline Colombia & 0 & 0 & 0 & 0 & 0 & 0 & 0 \\
\hline Libano & 0 & 0 & 0 & 0 & 1 & 0 & 0 \\
\hline Subtotal & 1.909 & 3.501 & 3.583 & 2.864 & 4.170 & 4.645 & 4.384 \\
\hline TOTAL & 1.961 & 3.626 & 3.606 & 2.999 & 5.242 & 5.598 & 4.560 \\
\hline
\end{tabular}

(Fuente: Foreing Agricultural Service, US Department of Agriculture) 
China es lejos el principal proveedor de piñones al mercado estadounidense, con una participación del $95 \%$ en el volumen total importado. Otros proveedores de cierta importancia son Afganistán, España, Hong Kong, Turquía y Pakistán (Cuadro $\mathrm{N}^{\circ} 5$ ). Sin embargo, lo notable es que la mayor parte de los piñones son recolectados en los bosques rusos y enviados a China para ser procesados, embalados y exportados hacia EEUU y otros paises importadores.

Bajo tal circunstancia, en una perspectiva de futuro hay dos aspectos fundamentales a considerar. Primero, las grandes extensiones de bosques rusos de Pinus sibirica y Pinus koraiensis están sometidos a una fuerte presión de explotación para la producción de madera, de tal manera que la superficie disponible se está reduciendo rápidamente. Además, de acuerdo a información de la WWF de Rusia, la explotación ilegal de pino de Siberia y pino de Corea alcanza al menos a unos 20 millones de $\mathrm{m}^{3}$ cada año, enviándose la mayoria de esta madera a China y Japón. Segundo, la industria rusa de piñones y sus productos ha crecido notablemente. con una integración vertical que va desde los recolectores y procesadores hasta los mayoristas, con un mercado local creciente que es mucho más atractivo que las exportaciones, con lo cual la disponibilidad de piñones rusos para ser exportados via China al mercado estadounidense se verá seriamente afectada.

En la Figura $\mathrm{N}^{\circ} 4$ se puede observar los canales de comercialización más tradicionales para que un kilo de piñones cosechado en Rusia llegue al consumidor final de EEUU. En cada canal se señala la agregación de valor correspondiente, destacándose el hecho de que el consumidor ruso tiene a su alcance un producto a un precio más de dos veces inferior al que debe pagar el consumidor estadounidense. La cadena de agregación de valor muestra que la producción de aceite y de diversos alimentos de piñones también es muy interesante para el mercado ruso.

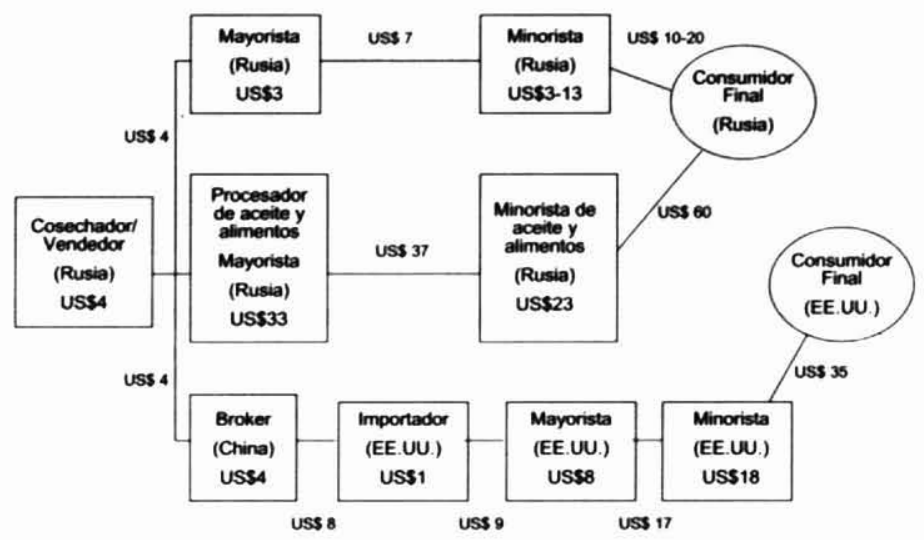

(Fuente: Sharashkin y Gold, 2004).

Figura $\mathrm{N}^{\circ} 4$

CANALES DE COMERCIALIZACIÓN PARA PIÑONES IMPORTADOS POR ESTADOS UNIDOS 


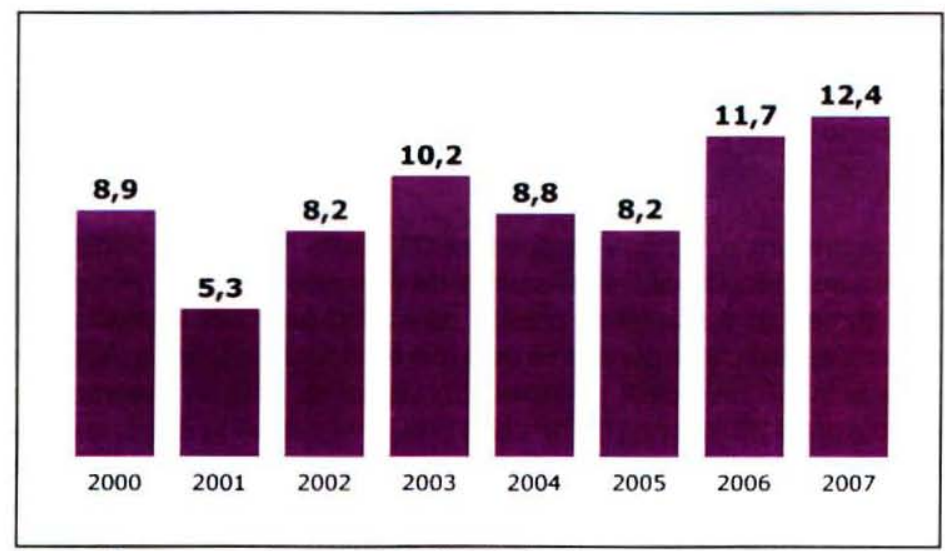

Fuente: Departamento de Comercio de Estados Unidos HS: 080290.25

Figura $\mathrm{N}^{\circ} 5$

\section{PRECIO PROMEDIO DEL PIÑÓN SIN CÁSCARA IMPORTADO EN ESTADOS UNIDOS (US\$/kilo)}

El interesante crecimiento de la demanda por piñones en EEUU y en el mundo en general, lleva a los especialistas de este pais a destacar la necesidad de abordar los siguientes temas (Sharashkin y Gold, 2004):

a) El replanteamiento de las prácticas de manejo forestal en tierras de bosques de pinos. donde se incorpore explicitamente el valor económico de la producción de piñones.

b) La investigación del potencial de producción de piñones a partir de otras especies nativas del género Pinus.

c) La introducción de especies productoras de piñones, nativas o exóticas, en los programas de plantaciones y en los sistemas agroforestales como árboles multipropósito.

Habria que agregar a lo anterior la posibilidad de importar piñones desde otros origenes distintos de China, entre los cuales Chile podria alcanzar un desarrollo interesante. por su cercania geográfica y comercial con el mercado de EEUU. 


\section{Europa}

En el mercado europeo existe una demanda importante por piñones del pino, en el cual actúan con mayor presencia los dos tipos de frutos que distingue el comercio de este producto: Los piñones chinos y los piñones del pino piñonero. El pino piñonero (Pinus pinea Linneo) es una especie tipicamente xerófila, propia del clima mediterráneo, especialmente extendida por la geografia española, plantado en formaciones naturales. Según datos de FAO, a nivel mundial abarca un área de $\mathbf{4 8 0}$ mil ha: $75 \%$ por ciento en España, 9\% en Portugal, $5 \%$ en Italia, esto es $89 \%$ en Europa, y $9 \%$ en Turquia. En España las masas principales se encuentran en la zona centro y norte del pais, siendo compleja la estimación de su superficie debido a que con frecuencia se encuentra compartiendo su dominancia, o como especie subordinada, con Quercus suber, Quercus ilex y Pinus pinaster, entre otras especies. En otros casos, forma pequeños bosquetes dificilmente cartografiables o inventariables.

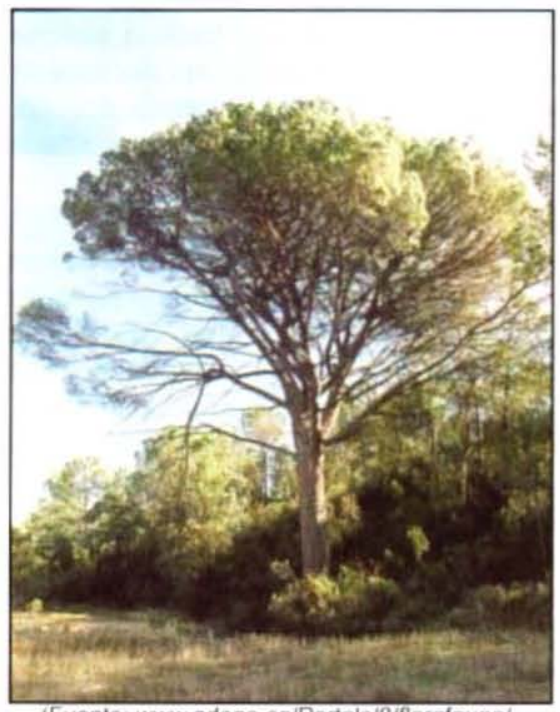

(Fuente: www adene es/Portals/8/florafauna/ ArbMon/pinocasasreig_p.jpg).

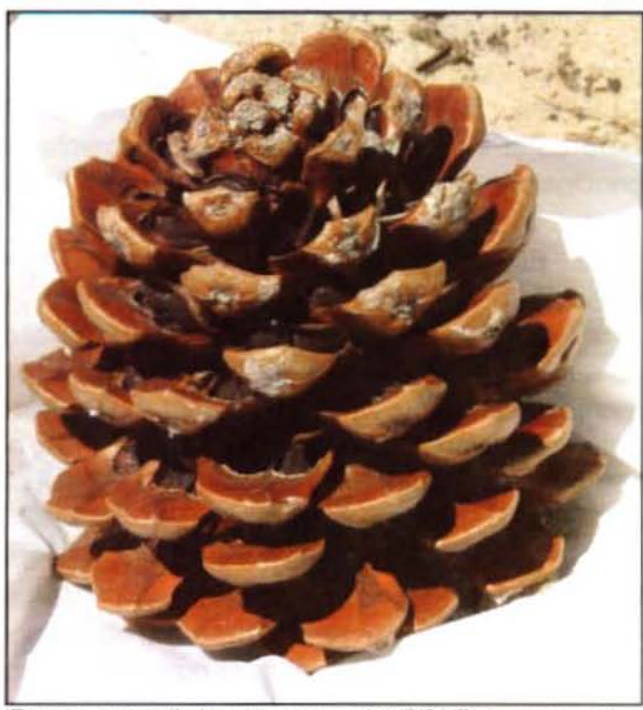

(Fuente www arikah.net/commons/en/6/61/Pine cone_with nuts.jpg).
Figura $\mathrm{N}^{\circ} 6$ ÁRBOL DE Pinus pinea
Figura $\mathrm{N}^{\circ} 7$

PIÑA DE Pinus pinea

España constituye el mayor productor mundial de piñón, representando aproximadamente el $45 \%$ de la producción total de piñones con cáscara, lo que supone unos 9 millones de euros. De las 30.000 t en las que se estima la producción mundial de piñon con cáscara, España. Portugal, Italia y Turquia son responsables de unas $25.000 \mathrm{t}$ anuales. De estos datos se deduce que España tiene una producción aproximada de 15.000 t de piñón con cáscara, lo que supone una producción de $1.000 \mathrm{t}$ anuales de piñón en blanco (sin cáscara). A esto hay que agregar 
la producción de piñones en blanco que España genera a partir de las $30.000 \mathrm{t}$ de piñas que importa desde Portugal, sin embargo, esta cifra no se conoce con exactitud (Sigfredo Ortuño, Universidad Politécnica de Madrid, pers. com.).

Según los datos del Ministerio de Medio Ambiente y Medio Rural y Marino de España, entre 1974 y 2000 la producción media anual de piñón con cáscara en España fue de $6.466 \mathrm{t}$, con un máximo de 10.360 t en 1981 y un mínimo de 3.201 t en 1984. Más aún, en el año 2003 la producción fue muy alta, con $11.156 \mathrm{t}$. Esta oscilación en la producción anual se debe a la importante variación en la productividad media, la que puede fluctuar entre 300 y $1.200 \mathrm{~kg} / \mathrm{ha} / \mathrm{año}$ de piñones con cáscara, con un rendimiento en piñón blanco de $17,5 \%-20 \%$. Esto hace que en algunos años se obtenga importantes beneficios, mientras que en otros periodos se puede llegar a grandes pérdidas, situación que se ha mantenido gracias a la estructura predominantemente familiar que gira en torno a este recurso y a la politica de ahorrar para soportar los años dificiles. propios de una economia de subsistencia (Barranco y Ortuño, 2004).

El análisis de los datos del mercado mundial del piñón refleja que España exporta aproximadamente 1.000 t de piñón en blanco a unos 21 paises, lo que supone un valor total de 10 millones de euros, frente a los 30 millones de euros que exporta china. Dentro de Europa, Italia es el mayor importador, principalmente de España, mientras que Francia se abastece principalmente de China y Alemania de Portugal (AFOVA, 2004).

El volumen de mercado anual de piñones en blanco, únicamente para estos tres paises (Italia, Francia y Alemania), es de unos 27 millones de euros, de los cuales España participa con 7 millones de euros, quedando un mercado potencial de aproximadamente 20 millones de euros más. Fuera de la Unión Europea, Estados Unidos constituye un gran importador con montos interesantes y crecientes, de los cuales una gran parte corresponden a piñón chino. Este antecedente es especialmente relevante, porque pone de manifiesto el gran potencial que el mercado norteamericano constituye para el piñón de España (AFOVA, 2004).

Con la excepción de los piñones de Pinus pinea, los precios que muestran los piñones de las diferentes especies comerciales no presentan gran diferencia en la oferta que se encuentra en Internet. No obstante, si existe una clara supremacia del precio del piñón de Pinus pinea, el que en promedio casi duplica el precio de su competencia. Posiblemente esta diferencia haya sido mayor en épocas pasadas, lo que sugiere un importante efecto de la presencia cada vez más agresiva del piñón asiático.

La gama de precios que ofrecen productores y distribuidores en las ofertas en línea muchas veces no se observa tan clara respecto del origen del piñón, de la misma manera como lo expresan clientes que han comprado piñones en tiendas del mercado norteamericano. A esto se agrega el desconocimiento y dificultad del consumidor común en diferenciar las caracteristicas y sabores de unos y otros, lo que finalmente se ha traducido en una pérdida de competitividad del piñón europeo.

En este sentido, es frecuente encontrar que el piñón chino se publicita en Internet como "Chinese Pignolias", "Pine Nuts Pignolias" o "Pignolias", este último término utilizado también para ofertar algunos piñones norteamericanos. Es decir, existe una mezcla de 
nombres comerciales para referirse al producto, lo que podria generar confusión al momento de diferenciar las procedencias por parte de los consumidores.

En el mercado europeo, especialmente en España, es clara la diferenciación que se realiza en cuanto al origen del producto, por la tradición histórica de ser un productor importante de piñones de la principal especie comercial. Sin embargo, los propios productores de piñones en Castilla y León, una de las zonas productoras más importantes, se enfrentan al problema de lidiar con precios finales excesivos cobrados por boutiques de alimentación y tiendas de delicatessen, a pesar de que los márgenes que ellos obtienen son bajisimos.
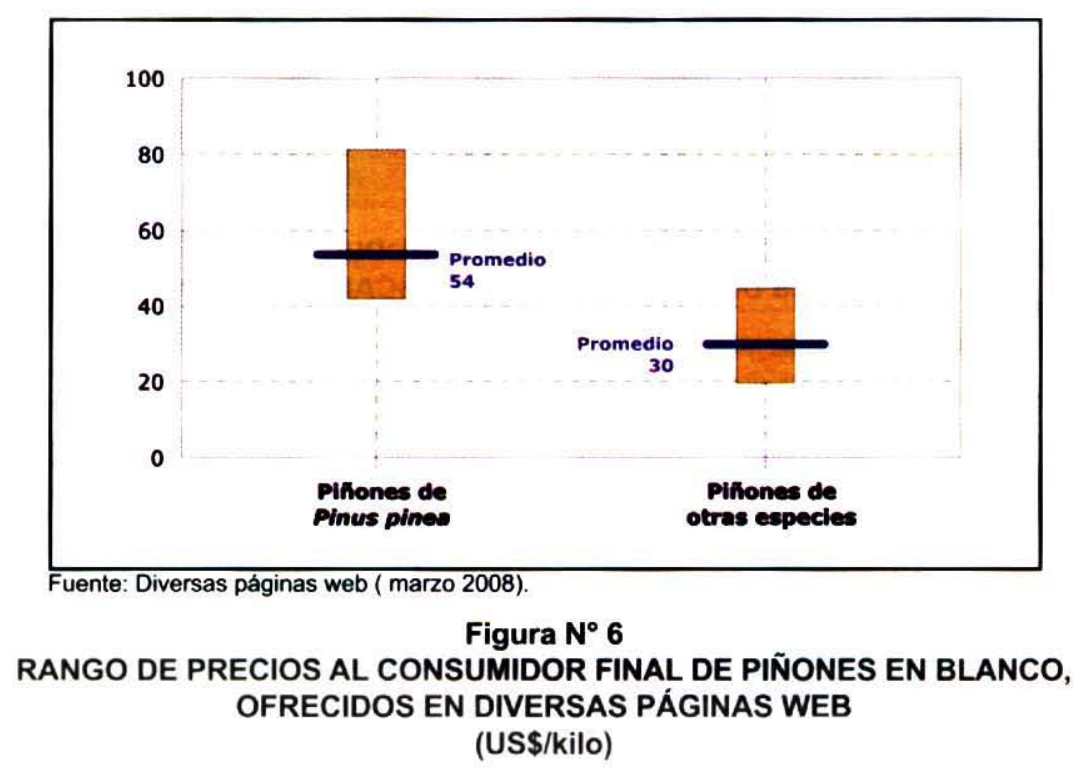


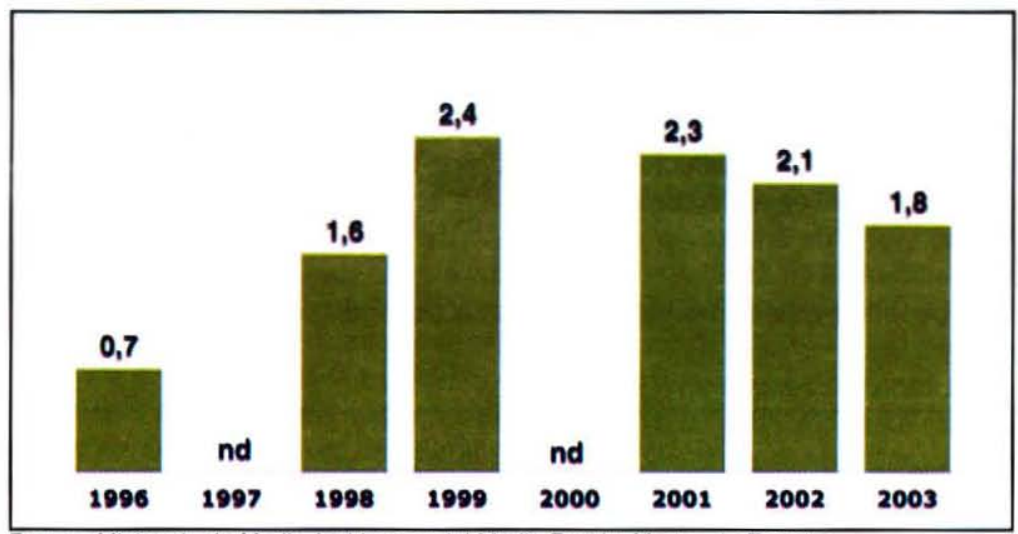

Fuente: Ministerio de Medio Ambiente y del Medio Rural y Marino de España

\section{Figura $\mathrm{N}^{\circ} 7$ \\ EVOLUCIÓN DEL PRECIO PRODUCTOR DE PIÑONES DE Pinus pinea CON CÁSCARA EN ESPAÑA (US\$/kilo)}

Los altos costos de los trabajos de recolección de la piña y de procesamiento del piñón, asi como el elevado precio de la piña entera que obtienen los productores, son los principales factores que afectan el negocio. Para enfrentar esta situación, los piñoneros de Castilla y León crearon en 2003 la primera Cooperativa Piñonera, cuyo objetivo es reducir los costos del procesamiento del piñón en blanco mediante un único proceso de descascarillado para todos los socios.

En 1996 en España, ICONA vendia semillas de pino piñonero a 860 pesetas/kg más el $7 \%$ de IVA (US\$ 7,1/kilo), mientras que en mercados de Valladolid el kilo de semillas limpias alcanzaba un valor de 4.000 pesetas, equivalentes a US\$30,8/kilo (INFOR, 1997). Actualmente, los precios del piñón en Europa fluctúan entre US\$20 y US\$31/kilo, incluso más, dependiendo del volumen de compra y en algunos casos por grados de calidad.

\section{ANTECEDENTES DE MERCADO EN CHILE}

En Chile, la comercialización de piñones de pino prácticamente no ha existido, situación que no debe extrañar puesto que muy pocos consumidores chilenos conocen sus usos y sus propiedades alimenticias. En los pocos casos que se ha detectado algún comercio de estos piñones, éstos generalmente se han dado en los negocios frecuentados por residentes de origen español o italiano. Este es el caso de la panaderia San Camilo, donde en 1996 se vendia piñones en paquetes de 100 gramos a \$2.150, lo que considerando el valor promedio del dólar para ese año ${ }^{5}$ equivale a US\$ 52/kilo (INFOR, 1998).

En los últimos años, ha habido algunas importaciones esporádicas por parte de

\footnotetext{
${ }^{3}$ Precio del dólar en 1996: \$ 412,27.
} 
empresas chilenas. Entre 2002 y 2007, se registraron sólo cuatro importaciones de piñones, dos en julio 2006, una en mayo 2007 y otra en octubre 2007, todas provenientes de Estados Unidos. El volumen total importado en este periodo fue de $231 \mathrm{~kg}$.

Tres de las partidas de importación fueron realizadas por el importador Proboka S.A., empresa nacional dedicada al rubro restaurantes y a la distribución de productos gourmet. Proboka es propietaria del restaurant de comida italiana "Geraldine" ubicado en el barrio alto de Santiago y especializado en comida internacional con énfasis en la italiana. La cuarta partida fue importada en octubre de 2007 por Comercializadora Global Trading Ltda., una empresa mayorista nacional del rubro alimentos.

\section{Cuadro $\mathrm{N}^{\circ} 6$ \\ DECLARACIONES DE IMPORTACIÓN DE PIÑONES DE PINO A CHILE}

\begin{tabular}{|c|c|c|c|c|}
\hline Fecha & Julio 2006 & Julio 2006 & Mayo 2007 & Octubre 2007 \\
\hline Importador & PROBOKA S.A. & PROBOKA S.A. & PROBOKA S.A. & $\begin{array}{l}\text { COMERCIAL } \\
\text { GLOBAL } \\
\text { TRADING LTDA. }\end{array}$ \\
\hline $\begin{array}{l}\text { Descripción del } \\
\text { producto }\end{array}$ & $\begin{array}{c}\text { PIÑONES DE } \\
\text { PINO, } \\
\text { EN BOLSAS DE } \\
2 \text { OZ }\end{array}$ & $\begin{array}{c}\text { PIÑONES } \\
\text { DE PINO, EN } \\
\text { BOLSAS DE } \\
5 \text { LB }\end{array}$ & $\begin{array}{c}\text { PIÑONES } \\
\text { DE PINO, EN } \\
\text { BOLSAS DE } 2 \text { OZ }\end{array}$ & $\begin{array}{c}\text { PIÑONES DE } \\
\text { CÁSCARA, EN } \\
\text { CAJAS DE CARTON }\end{array}$ \\
\hline Marca & ALESSI & VIGO & ALESSI & AL NOUR \\
\hline $\begin{array}{l}\text { Pais } \\
\text { origen }\end{array}$ & $\begin{array}{l}\text { ESTADOS } \\
\text { UNIDOS }\end{array}$ & $\begin{array}{l}\text { ESTADOS } \\
\text { UNIDOS }\end{array}$ & $\begin{array}{l}\text { ESTADOS } \\
\text { UNIDOS }\end{array}$ & ESTADOS UNIDOS \\
\hline Código arancel & 0802.9000 & 0802.9000 & 0802.9000 & 0802.9000 \\
\hline $\begin{array}{l}\text { Cantidad } \\
(\mathrm{kg})\end{array}$ & 3,6 & 68.1 & 10,7 & 148,1 \\
\hline $\begin{array}{l}\text { Valor CIF } \\
\text { (US\$) }\end{array}$ & 104,5 & 977,4 & 320,5 & 874,2 \\
\hline $\begin{array}{l}\text { Precio CIF } \\
\text { (US\$/kg) }\end{array}$ & 29,0 & 14,4 & 30,0 & 5,9 \\
\hline
\end{tabular}

(Fuente: INFOR, con datos de Aduana).

En el Cuadro $\mathrm{N}^{\circ} 6$ es posible observar, en primer lugar, que no se hace mención de la especie en la descripción de la mercancia, estimándose que se debe tratar de piñones norteamericanos o asiáticos. En segundo lugar, si se considera el precio por kilo, se advierte que éste es muy superior en envases de $2 \mathrm{oz}(5,6 \mathrm{~g})$ que en los de 5 libras $(2,27 \mathrm{~kg})$, posiblemente por el mayor valor que significa comercializar en menores cantidades, por ejemplo, por los tamaños, calidades y caracteristicas de los envases utilizados. Para las bolsas de $2 \mathrm{oz}$, la equivalencia en moneda nacional, expresada en kilos, registró en esta importación un precio promedio de $\$ 15.668$ por kilo ${ }^{6}$, mientras que para el producto presentado en bolsas de 5 libras

\footnotetext{
${ }^{6}$ Promedio del embarque de julio 2006 (\$540,62/dólar) y de mayo 2007 (\$522,02/dólar).
} 
el precio fue de $\$ 1.940^{7}$.

Considerando que estos precios son los pagados por el importador local, el precio que finalmente se le da al consumidor final evidentemente que se verá incrementado, aspecto que de alguna forma valida la dinámica de incremento de precios en la cadena de comercialización ilustrada para las importaciones de piñones en Estados Unidos.

\section{ZONAS POTENCIALES PARA EL ESTABLECIMIENTO DEL PINO PIÑONERO EN CHILE}

En 1997, INFOR determinó la superficie potencial para el establecimiento de Pinus pinea en Chile entre las regiones VI y IX del pais en el marco del Proyecto "Potencialidad de especies y sitios para una diversificación silvicola nacional". En este estudio se consideró todos los requerimientos edáficos y climáticos, lo que permitió determinar un total cercano a 1,4 millones de hectáreas potenciales. De esta superficie, la mayor proporción se encuentra ubicada en la Región del Maule.

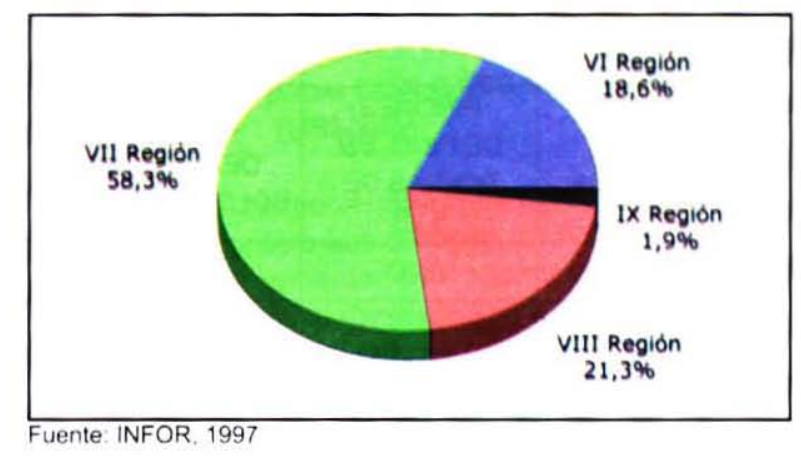

\section{Figura $\mathrm{N}^{\circ} 8$ \\ SUPERFICIE POTENCIAL REGIONAL PARA EL ESTABLECIMIENTO DE Pinus pinea EN CHILE}

(Total Nacional: 1.397 .898 hectáreas)

Cabe señalar que esta superficie sólo se considera indicativa de la potencialidad de establecimiento de la especie y no como superficie útil, puesto que no está corregida por restricciones como uso de la tierra, capacidad de uso del suelo, áreas silvestres protegidas. bosque nativo e infraestructura, entre otras. Un estudio que incluya estas restricciones, asi como una escala superior de análisis en términos cartográficos, proporcionará información con mayor precisión.

En el mismo proyecto mencionado anteriormente, INFOR evaluó económicamente las plantaciones de Pinus pinea bajo distintos esquemas de manejo y escenarios de costos y precios. llegando a determinar que en la mayoria de las opciones, que abarcaban un amplio rango de situaciones, las rentabilidades eran positivas. Aunque dentro de las alternativas

\footnotetext{
${ }^{7}$ Precio del dólar en julio 2006. \$540.62.
} 
evaluadas se incorporó la bonificación forestal, cabe señalar que esta especie no ha gozado de tal beneficio, debido a la baja densidad de plantación que se requiere para favorecer la obtención de frutos.
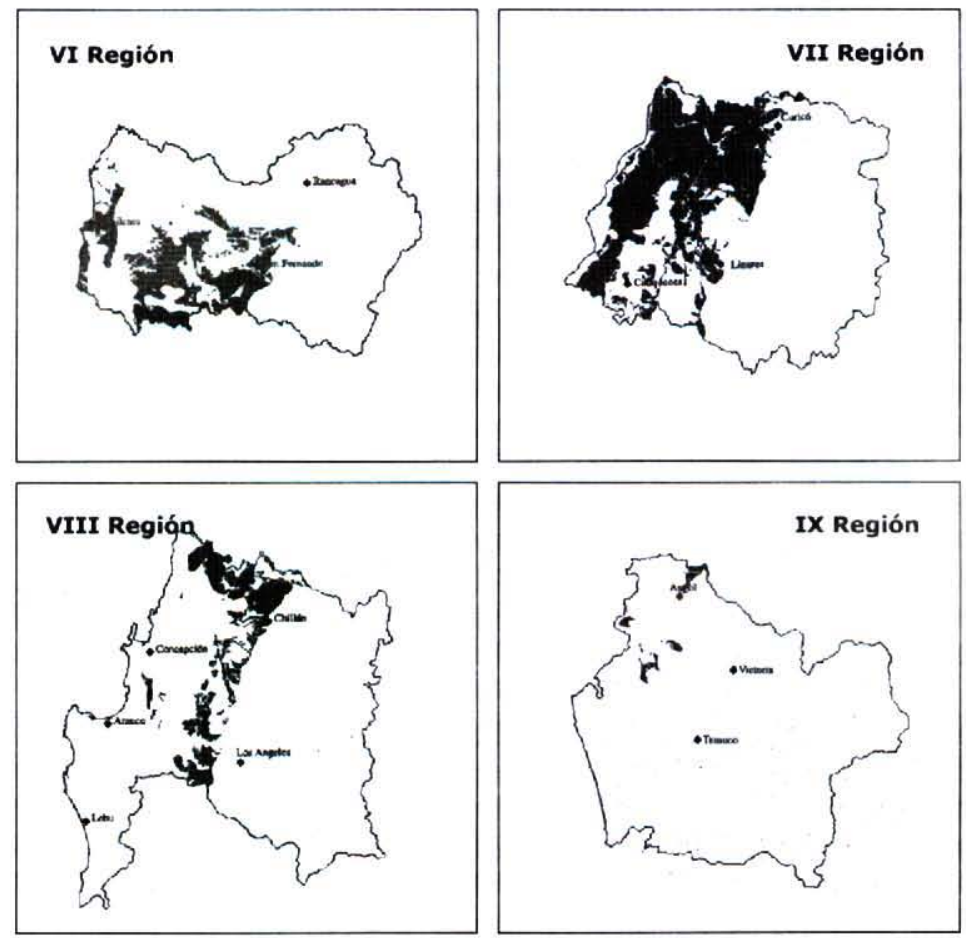

(Fuente: INFOR, 1997)

Figura $\mathrm{N}^{\circ} 9$

ZONAS POTENCIALES REGIONALES PARA EL ESTABLECIMIENTO DE Pinus pinea EN CHILE

Por sus condiciones de cultivo fruto-forestal y los conocimientos que se tiene en la actualidad del desarrollo de la especie en Chile, deben destacarse los siguientes aspectos:

a) Pinus pinea no requiere de sitios exigentes (menor costo).

b) Los retornos comienzan al año 15 para luego crecer en forma permanente, representando para el inversionista un ingreso anual a partir de ese año. Sin embargo, éste podría adelantarse hasta los 5 u 8 años, acentuando el atractivo de este cultivo.c) c) Las características del cultivo lo hacen muy apropiado para pequeños y medianos propietarios, que podrian alcanzar rápidamente una masa crítica plantada, especialmente mediante la asociatividad. 
Actualmente se están actualizando las áreas potenciales de la especie, considerando nueva información y antecedentes biológicos y de cultivo de la especie recogiendo la experiencia de los paises que más trabajan con la especie.

\section{OTROS ANTECEDENTES}

Es importante señalar que resulta dificil dimensionar el mercado internacional de los piñones, porque no todos los paises tienen una glosa arancelaria especifica que los identifique, de modo que cuando los volúmenes son bajos, las importaciones y exportaciones se informan en forma conjunta con otros frutos secos. En este caso, la glosa 080290 corresponde a frutos secos y es universal. Con dos digitos más, el código del Sistema Arancelario identifica el tipo de fruto seco, pero éste ya no es universal de tal manera que cambia entre paises o bloque comercial. En el caso de China es el 08029030 y en EEUU se utiliza el 08029020 (piñones sin pelar) y 08029025 (piñones pelados). En España, y en toda la Unión Europea, el código utilizado para el comercio exterior de piñones es el 08029050.

De acuerdo con Ortuño $(2008)^{8}$, las estadisticas de comercio exterior de piñones que registra la Unión Europea son extremadamente confusas, pues no reflejan el verdadero comercio del piñón pelado. Esto, debido a que las piñas del pino también son registradas en la glosa 080290. En efecto, las piñas son un producto que se comercializa muy frecuentemente entre los principales paises productores de piñones de Europa (España, Portugal, Italia y otros), y alcanzan precios muy diferentes al piñón pelado. De esta forma, los volúmenes y montos registrados en Eurostat en la glosa arancelaria para piñón, muestra enormes diferencias entre paises y entre periodos. Esta situación es especialmente evidente para España.

Por otra parte, un aspecto interesante es que actualmente el principal productor mundial de piñones de pino es China, pais que sólo tiene una trayectoria de 25 años en este producto. Sus piñones son de forma triangular y su sabor es menos nitido que los producidos en Europa. Los piñones mediterráneos son más delgados y elongados, con un pronunciado sabor tipo nuez; son considerablemente más caros que los piñones chinos (dos o tres veces más), a pesar de lo cual son preferidos por los chefs y pasteleros (Epicurean On Line).

En EEUU, gran importador de piñones de China, el precio de venta a minoristas se ha mantenido estable durante los últimos años, a pesar de que los precios de importación han experimentado variaciones significativas. De hecho, hace unos pocos años China tuvo una gran producción que hizo bajar los precios, sin embargo, el precio al consumidor en el mercado estadounidense no cambió. Esto indica que el mercado de importación no tiene mayor influencia en el mercado interno de este pais, lo cual se explica porque los piñones de pino son un producto muy apreciado y relativamente escaso. En efecto, comercializadores de distintas partes del pais (Los Ángeles, New York y Virginia, por ejemplo) tienen dificultades para encontrar suficientes piñones que les permitan abastecer los gigantescos pedidos del sector alimentario, destinados principalmente a la elaboración de pesto congelado, pizza de pesto y ensalada de pasta con pesto, alimentos que se venden explicitando el contenido de piñones (Epicurean On Line).

\footnotetext{
${ }^{\circ}$ Comunicación personal
} 
Por último, cabe señalar que Nueva Zelanda también se ha convertido en uno de los grandes importadores de piñones, puesto que se usan ampliamente para cocinar, en ensaladas, pasteleria y como fruto seco de consumo directo.

\section{CONCLUSIONES}

Las estadísticas de comercio internacional de piñones son dificiles de consolidar debido a las diferentes posiciones arancelarias de cada país y a que frecuentemente se agrupan piñones pelados y no pelados. Incluso, en algunos paises de Europa, se comercializan las piñas del pino dentro de estos códigos arancelarios, lo que conduce a interpretaciones erróneas en los montos y cantidades que se transan y, más aún, en los precios estimados.

Estados Unidos es el principal importador mundial de piñones, fundamentalmente de piñón chino, en cantidades que bordean las 5 mil toneladas anuales. En Europa, Italia, Francia y Alemania son los principales importadores, tanto de piñón de Pinus pinea como del piñón chino. Por su parte, España es el mayor productor y exportador de piñones de Pinus pinea.

Respecto de los precios, se constató que en una rápida revisión de diversas páginas web que ofrecen piñones, el precio del piñón europeo (Pinus pinea) supera en cerca de $80 \%$ al precio de su competidor directo, el piñón chino. Cabe destacar que este último ha comenzado a tener cada vez mayor presencia en el contexto mundial, por lo que esta brecha de precios pudiera acortarse, situación que incluso es advertida por los productores españoles.

En Chile existe potencialidad para el establecimiento de Pinus pinea. INFOR determinó una superficie potencial de 1,4 millones de hectáreas entre las regiones de $\mathrm{O}^{\prime}$ Higgins y la Araucania, realizando además estudios de rentabilidad de diversas alternativas, la mayoría de las cuales resultaron positivas y muy atractivas para los pequeños y medianos propietarios. Es recomendable realizar nuevos estudios que entreguen las superficies útiles a plantar por cada región e incluso sentar las bases para una futura inclusión de la especie dentro de la actual bonificación forestal.

Además, se hace necesario realizar estudios acabados sobre las propiedades de los piñones que crecen en Chile, tanto para su introducción en el mercado local como para analizar las perspectivas de exportación futura.

En cuanto al mercado local, se sugiere potenciar su ingreso en el segmento gourmet, asi como realizar degustaciones y diversificar las preparaciones alimenticias, entre otros, focalizándose en los potenciales clientes consumidores, como restaurantes y tiendas especializadas en frutos secos.

También se sugiere investigar las posibilidades de exportar hacia el mercado europeo y de EEUU, analizando la rentabilidad de un proyecto de establecimiento de plantaciones de Pinus pinea, cuyo principal producto sea el piñón. En este sentido, es probable que la experiencia de rápido crecimiento de este rubro en China, sea una buena lección a estudiar.

Con todo, se concluye que desarrollar un segmento de producción de piñones de pino 
en Chile generará una actividad innovadora en muchos aspectos, que puede ser altamente rentable y representar una fuente laboral para un número significativo de personas, desde el bosque hasta los locales comerciales y restaurantes. 


\section{REFERENCIAS}

AFOVA, 2004. El pino piñonero es España, importancia, necesidades y potencialidad. Asociación Forestal de Valladolid (AFOVA).

<http://www.agrodigital.com/PIArtStd.asp?CodArt=33748> [consulta: Abril 2008]

Barranco, J; Ortuño, S. 2004. Aproximación del sector del piñón en España. En Revista española de estudios agrosociales y pesqueros. 2004,201.

$<$ http://www.mapa.es/es/ministerio/pags/Biblioteca/revistas/rev_numero.asp> [consulta: Abril 2008]

Epicurean On Line. Pine Nuts - The Popularity of Pignoli by Janet Fletcher.

$<$ http://www.epicurean.com/articles/pine-nuts-pignoli.html> [consulta: Abril 2008]

FAO, 1995. Non-wood forest products from coniferous. Forestry Department, Food and Agricultural Organization of the United Nations.

<http://www.fao.org/docrep/X0453E/X0453e00.htm> [consulta: Abril 2008]

Foreing Agricultural Service. United States Department of Agriculture.

$<$ http://www.fas.usda.gov/> [consulta: Abril 2008]

INFOR-CONAF, 1997. Monografia del Pino piñonero, Pinus pinea. Instituto Forestal Corporación Nacional Forestal. 100 p.

Ministerio de Medio Ambiente y Medio Rural y Marino de España, 2006. Anuario de Estadistica Agroalimentaria 2006.

<http://www.mapa.es/es/estadistica/pags/anuario/introduccion.htm\#art2> [consulta: Abril 2008]

Ortuño, Sigfredo. Profesor del Departamento de Economia y Gestión Forestal, de la Escuela Técnica Superior de Ingenieros de Montes, Universidad Politécnica de Madrid.

Salas-Salvadó, J; Ros, E; Sabaté, J. 2005. Frutos secos, salud y culturas mediterráneas. <http://books.google.cl/books? id=T6CWdFYcYskC\&dq=comercio+mundial+frutos+secos\&sou rce=gbs_summary_s\&cad $=0>$ [consulta: Abril 2008]

Sharashkin, L; Gold M. 2004. Pine Nuts (Pignolia): Species, Products, Markets, and Potential for U.S. Production. In: Northern Nut Growers Association 95th Annual Report. Proceeding for the 95th annual meeting, Columbia, Missouri, August 16-19, 2004.

$<$ http://www.pinenut.com/noha.htm> [consulta: Abril 2008] 